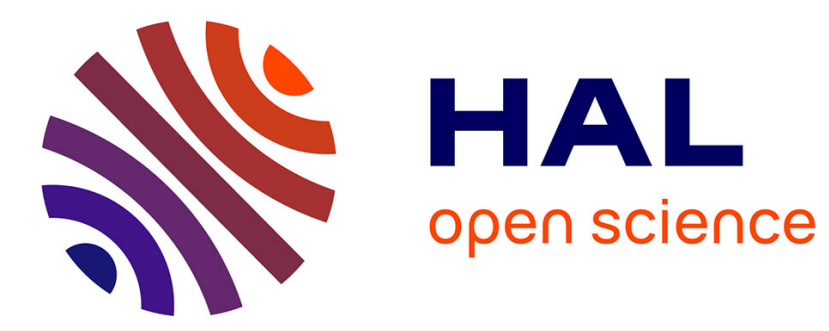

\title{
EXPANSION IN MOMENTS AND DISORDERED SYSTEMS
}

\author{
F. Cyrot-Lackmann
}

\section{To cite this version:}

F. Cyrot-Lackmann. EXPANSION IN MOMENTS AND DISORDERED SYSTEMS. Journal de Physique Colloques, 1974, 35 (C4), pp.C4-109-C4-114. 10.1051/jphyscol:1974419 . jpa-00215610

\section{HAL Id: jpa-00215610 https://hal.science/jpa-00215610}

Submitted on 1 Jan 1974

HAL is a multi-disciplinary open access archive for the deposit and dissemination of scientific research documents, whether they are published or not. The documents may come from teaching and research institutions in France or abroad, or from public or private research centers.
L'archive ouverte pluridisciplinaire HAL, est destinée au dépôt et à la diffusion de documents scientifiques de niveau recherche, publiés ou non, émanant des établissements d'enseignement et de recherche français ou étrangers, des laboratoires publics ou privés. 


\title{
EXPANSION IN MOMENTS AND DISORDERED SYSTEMS
}

\author{
F. CYROT-LACKMANN
}

\author{
Institut Laue-Langevin, B.P. 156, 38042 Grenoble Cedex, France
}

\begin{abstract}
Résumé. - On montre que la technique de développement en moments de la densité d'états est un outil très utile pour l'étude des systèmes désordonnés. Les moments peuvent être calculés directement comme une fonction des intégrales de recouvrement et de l'ordre local caractérisant le système. Parmi les techniques possibles pour obtenir la densité d'états à partir de la seule connaissance de ses premiers moments, la meilleure repose sur un développement en fraction continue de sa transformée de Hilbert. Cette méthode est brièvement discutée, et l'on montre comment des informations précises peuvent être obtenues sur d'éventuelles bandes d'énergie interdite ou d'éventuels points critiques dans la densité d'états.

On donne ensuite des exemples des applications possibles de la méthode des moments à différents cas de systèmes désordonnés. Dans le cas des systèmes à désordre de composition, c'est-àdire par exemple des alliages désordonnés, cette méthode permet une nouvelle simple déviation de l'approximation de potentiel cohérent, et peut être utilisée pour obtenir des résultats étendant cette approximation.

Dans le cas des systèmes avec désordre topologique, c'est-à-dire par exemple les métaux liquides ou les bandes d'impuretés, on donne des densités d'états détaillées, en utilisant en particulier une approche de simulation de ces systèmes sur ordinateur.

Abstract. - Expansion of the density of states in its moments is shown to provide a useful tool in the study of the electronic properties of tightly bound disordered systems. The moments can be directly computed as a function of the overlap integrals and the local order of the system. Among the possible techniques for obtaining the density of states from its low order moments, the best one, using a continued fraction expansion of its Hilbert transform, is briefly discussed. It is also shown how information about the possible band gaps and the remnants of critical points structure can be obtained through this technique. Examples of the possible applications of the moment method to various cases of disordered systems are then given. For systems with compositional disorder, i.e. disordered alloys, this approximation which provides a new simple derivation of the coherent potential approximation (CPA), can be used to obtain results extending the CPA. For systems with topological disorder, i.e. impurity bands of liquid metals, detailed density of states are given, using in particular a simulation of the random system on the computer.
\end{abstract}

1. Introduction. - This paper concerns a method recently developed to study the electronic properties of tightly bound disordered systems. Much research has been performed in this domain, where great difficulties arise due to the breakdown of the usual approach based on the periodicity of the lattice. Most of the results obtained are derived for one-dimensional disordered systems [1], but unfortunately neither the methods nor the results can in general be extended to the three-dimensional case.

The method presented here, based on an expansion of the electronic density of states in its moments, seems to be quite successful for deriving all possible information on the density of states of a three-dimensional disordered system and related physical properties. Indeed, in a tight binding scheme, the calculation of the moments of the density of states can be made directly, without reference to the real electronic states and in ordered as well as in disordered systems.

The moment expansion technique can be used in two different ways. By considering the asymptotic properties of the moments, one can get results on the limits of the density of states, and on its shape near these limits $[2,3]$. But, in practice, in disordered systems, it is usually possible to calculate exactly only the first moments. So, one has to use a technique to estimate the density of states from its low-order moments. Several methods have been used, but the one which seems the most precise and powerful as shown by a detailed comparison [4], is based on a continued fraction expansion of the Hilbert transform of the density of states.

In a first part, we describe the general features of the moment expansion technique. Then, we describe the continued fraction method for deriving the density of states from the low-order moments. In particular, we analyze the effect of band edge singularities or internal singularities and of band gaps in the coefficients of the continued fraction. In the last part, examples of the possible applications of the moment method to various cases of disordered systems are given. Special attention is given to problems involving diagonal disorder, such as random substitutional alloys.

2. Moments of the density of states. - Let us just briefly recall the main results of a moment expansion technique, showing in particular that the moments 
can be computed directly, as a function of the overlap integrals and the correlation functions characterizing the local order of the system.

Consider a one electron hamiltonian describing a narrow band made up of orbitals from $N$ atoms,

$$
\mathscr{H}=T+\sum_{i=1}^{N} V\left(\mathbf{r}-\mathbf{R}_{i}\right)=T+\sum_{i} V_{i} .
$$

The density of states per atom is :

$$
n(E)=\frac{1}{N} \operatorname{Tr} \delta(E-\mathscr{H}) .
$$

Its moment of order $p$ is :

$$
\mu_{p}=\int E^{p} n(E) \mathrm{d} E=\frac{1}{N} \operatorname{Tr} \mathfrak{H}^{p} .
$$

Calculating the moments as trace, any basis can be used. In particular, in a tight binding scheme the band being described as a linear combination of a set of atomic orbitals $\varphi_{i}$ centred on all the atomic sites, this atomic wave functions basis can be used :

$$
\left(T+V_{i}\right) \varphi_{i}=E_{0} \varphi_{i}
$$

where $E_{0}$ is the energy of the isolated atom. Keeping only the two centres overlap integrals,

$$
\begin{aligned}
& \alpha_{i}=\left\langle\varphi_{i}\left|\sum_{j \neq i} V_{j}\right| \varphi_{i}\right\rangle \\
& \beta_{i j}=\left\langle\varphi_{i}\left|V_{i}\right| \varphi_{j}\right\rangle
\end{aligned}
$$

and taking $E_{0}$ as the zero energy, the moment of order $p$ can be written :

$$
\mu_{p}=\sum_{i_{1} \ldots i_{p}} \gamma_{i_{1} i_{2}} \gamma_{i_{2} i_{3}} \ldots \gamma_{i_{p} i_{1}}
$$

where $\gamma_{i j}$ is either $\beta_{i j}$ or $\alpha_{i} \delta_{i j}$.

In disordered systems, we are interested by physical quantities averaged over all possible configurations of the system. Denoting this ensemble average process by brackets, we have for the mean density of states :

$$
<n(E)>=\int \underline{P}\left\{R_{i}\right\} n\left(E,\left\{R_{i}\right\}\right) \mathrm{d}\left\{R_{i}\right\}
$$

where $\underline{P}\left\{R_{i}\right\}$ is the density matrix giving the probability of having a configuration $\left\{R_{i}\right\}$. The computation of the successive moments $\left\langle\mu_{p}\right\rangle$ of the mean density of states needs the knowledge of the successive correlation functions $g^{(p)}\left(\mathbf{R}_{1}, \ldots, \mathbf{R}_{p}\right)$ giving the probability of having a set of $p$ atoms located in $\mathbf{R}_{\mathbf{1}}$, $\mathbf{R}_{2}, \ldots, \mathbf{R}_{p}$ :

$$
\begin{aligned}
g^{(p)}\left(\mathbf{R}_{1}, \ldots, \mathbf{R}_{p}\right)= & \\
& =\frac{N !}{N-p ! \rho^{p}} \int \underline{P}\left\{R_{i}\right\} \mathrm{d}^{3} \mathbf{R}_{p+1} \ldots \mathrm{d}^{3} \mathbf{R}_{N}
\end{aligned}
$$

where $\rho$ is the density of the system. Neglecting the $\alpha$-type overlap integrals for simplicity, then :

$$
\begin{array}{r}
<\mu_{p}>=\int \beta\left(\mathbf{R}_{1}, \mathbf{R}_{2}\right) \beta\left(\mathbf{R}_{2}, \mathbf{R}_{3}\right) \ldots \beta\left(\mathbf{R}_{p}, \mathbf{R}_{1}\right) \times \\
\times \tilde{g}^{(p)}\left(\mathbf{R}_{1}, \ldots, \mathbf{R}_{p}\right) \mathrm{d}^{3} \mathbf{R}_{1} \ldots \mathrm{d}^{3} \mathbf{R}_{p}
\end{array}
$$

where

$$
\begin{aligned}
& \tilde{g}^{(p)}\left(\mathbf{R}_{1}, \ldots, \mathbf{R}_{p}\right)= \\
&=g^{(p)}\left(\mathbf{R}_{1}, \ldots, \mathbf{R}_{p}\right)+\rho \delta_{\mathbf{R}_{1} \mathbf{R}_{2}} g^{(p-1)}\left(\mathbf{R}_{1}, \mathbf{R}_{3}, \ldots, \mathbf{R}_{p}\right)+ \\
&+\rho \delta_{\mathbf{R}_{1} \mathbf{R}_{3}} g^{(p-1)}\left(\mathbf{R}_{1}, \mathbf{R}_{2}, \mathbf{R}_{4}, \ldots, \mathbf{R}_{p}\right)+\cdots \\
&+\rho^{p} \delta_{\mathbf{R}_{1} \mathbf{R}_{2}, \ldots, \mathbf{R}_{p}} .
\end{aligned}
$$

For example, applying eq. (9), the first few moments are given by

$$
\begin{aligned}
&<\mu_{1}>=0 \\
&<\mu_{2}>=\rho^{2} \int g^{(2)}\left(\mathbf{R}_{1}, \mathbf{R}_{2}\right) \beta^{2}\left(\mathbf{R}_{1}, \mathbf{R}_{2}\right) \mathrm{d}^{3} \mathbf{R}_{1} \mathrm{~d}^{3} \mathbf{R}_{2} \\
&<\mu_{3}>=\rho^{3} \int g^{(3)}\left(\mathbf{R}_{1}, \mathbf{R}_{2}, \mathbf{R}_{3}\right) \beta\left(\mathbf{R}_{1}, \mathbf{R}_{2}\right) \beta\left(\mathbf{R}_{2}, \mathbf{R}_{3}\right) \times \\
& \times \beta\left(\mathbf{R}_{3}, \mathbf{R}_{1}\right) \mathrm{d}^{3} \mathbf{R}_{1} \mathrm{~d}^{3} \mathbf{R}_{2} \mathrm{~d}^{3} \mathbf{R}_{3} .
\end{aligned}
$$

Knowing the local range order characterizing the system, the moments can then be computed, either through eq. (9), or directly through (3) by generating the random system on a computer.

The density of states is uniquely determined by the knowledge of all its moments, through :

$$
n(E)=\frac{1}{2 \pi} \int_{-\infty}^{\infty} \mathrm{e}^{i x E}\left[\sum_{p=0}^{\infty} \frac{(-i x)^{p}}{p !} \mu_{p}\right] \mathrm{d} x
$$

Using general properties of Fourier transforms, it can easily be shown that asymptotic properties of the moments are directly related to band edges or internal singularities in the density of states $[2,5]$. This is of great application in disordered crystalline materials, such as crystals with extended defects or surface where the asymptotic part of the moments can be easily computed [5]. In more disordered systems, with compositional or translational disorder, this property is of more limited help as the high order moments can usually be calculated only through approximations [2].

3. Continued fraction expansion. - In disordered systems, one has to use the best approach to the finite problem of moments, i. e. to the problem of building up a function only through its first moments. Two different approaches can be taken. One can try to fit an a priori chosen function with the known moments, giving a semi-quantitative idea of the behaviour of the function. Or one can aim for exact results on the bounds of the integrated density of states, and then determine the best density of states. A detailed comparison of these various methods [4] shows that the more powerful method is based on expansion of the Hilbert transform $G(z)$ of the density of states

$$
G(z)=\int_{-\infty}^{\infty} \frac{n(E)}{z-E} \mathrm{~d} E
$$


into a continued fraction [6]

$$
G(z)=\frac{\alpha_{1}}{\alpha_{2}+z-\frac{\alpha_{2} \alpha_{3}}{\alpha_{3}+\alpha_{4}+z-\alpha_{4} \alpha_{5} \ldots}} .
$$

The coefficient $\alpha_{k}$ is a function of the $k$ first moments, $\mu_{0}, \mu_{1}, \ldots, \mu_{k-1}[4,5,7]$. These coefficients $\alpha_{k}$ are very rapidly converging towards their asymptotic value and the way they converge can be simply related to the properties of the density of states (possible singularities and band gaps). Indeed, let us put

$$
\begin{aligned}
& a_{n}=\alpha_{2 n-1}+\alpha_{2 n} \\
& b_{n}=\alpha_{2 n} \alpha_{2 n+1} .
\end{aligned}
$$

For a symmetric band, $\lim _{n \rightarrow \infty} a_{n}$ give the middle of the band. In the absence of band gap, the bandwidth $w$ is given by

$$
\lim _{n \rightarrow \infty} b_{n}=\frac{1}{16} w^{2} .
$$

The behaviour of the density of states near its edges can be shown to be related to the asymptotic behaviour of $b_{n}$ [4]. In particular, when the band shows internal singularities, the coefficients $b_{n}$ are decreasing in oscillating with a frequency connected to the position of the singularity and a damping function related to the nature of the singularity. The criterion (14) has to be compared with the corresponding criterion on the moments

$$
\lim _{n \rightarrow \infty} \frac{\mu_{2 n}}{\mu_{2 n-2}}=E_{b}^{2}
$$

where $E_{b}$ is the abscissa of the band edge the most distant from the origin. The coefficients $\alpha_{k}$ converge much more quickly than the moments, and so they can be continued with a minimum error. Thus, one determines the first $p$ coefficients from the knowledge of the first $p$ moments. Then, one extrapolates the $\alpha_{k}$ coefficients up to a higher level $(\simeq 200)$ by a constant function oscillating or not depending on the case

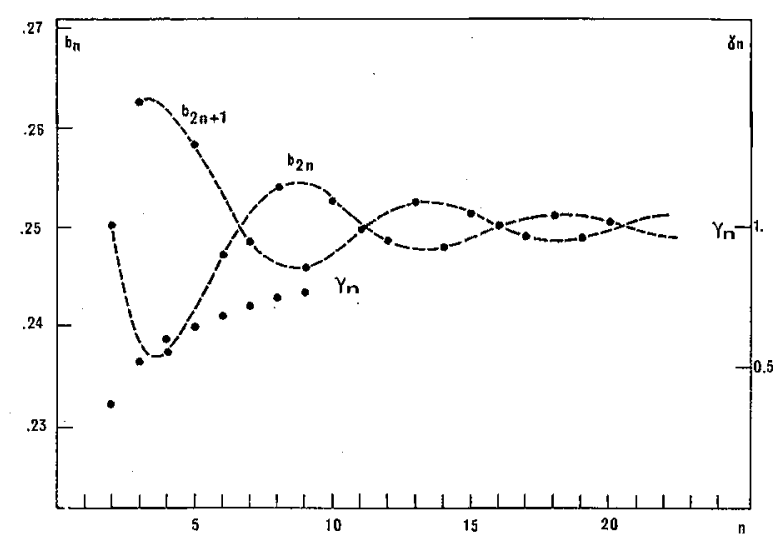

FIG. 1. - Plot of the coefficients $b_{n}$ and $\gamma_{n}=\mu_{2 n} / \mu_{2 n-2}$ for the simple cubic crystal. The coefficients $a_{n}$ are equal to zero. studied, so as to approach the Hilbert transform by a truncated fraction of $P$ levels. One can remark that $G(z)$ reduces then to a sum of single fractions

$$
G(z)=\sum_{i=1}^{p} \frac{w_{i}}{z-E_{i}}
$$

which is equivalent to approach the integrated density of states by a histogram of $P$ jumps.

For example, figures 1 and 2 show the result obtained with 14 moments for a simple cubic band, the unknown coefficients being continued either by a constant or by an oscillating function.

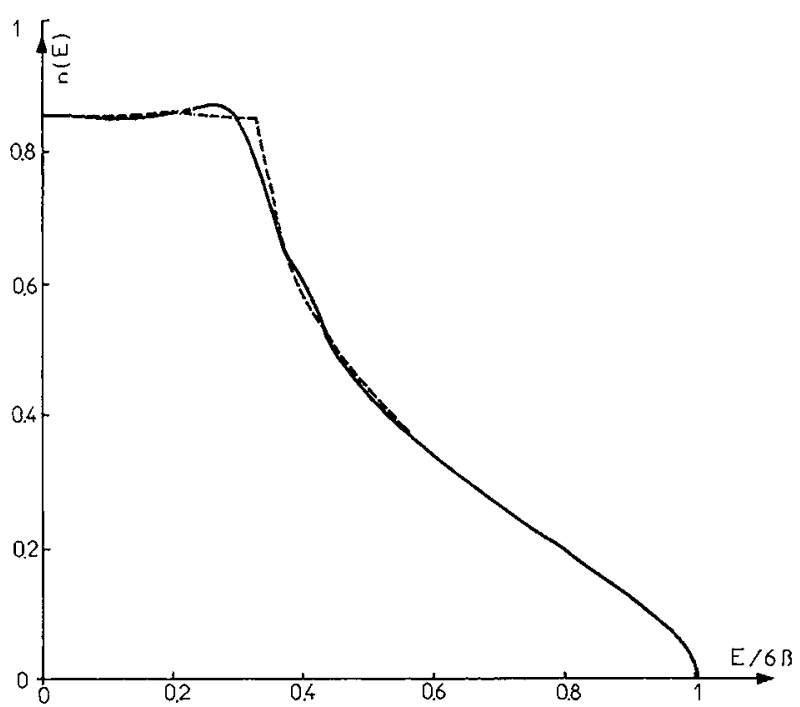

Frg. 2. - Density of states for the simple cubic crystal obtained by the continued fraction method with 14 moments. The coefficients $b_{n}$ are continued by a constant (dotted line) or by a damped oscillating function (solid line).

A similar analysis can be made when a gap $\Delta$ appears inside the band; indeed, the coefficients $b_{n}$ oscillate between two limits, $\bar{b}$ and $b$ such that

$$
\begin{aligned}
& \bar{b}=\frac{1}{16}(w+\Delta)^{2} \\
& b=\frac{1}{16}(w-\Delta)^{2} .
\end{aligned}
$$

This property can be of wide use to study the case of either random binary alloys or disordered covalent materials [8], where a gap may open in the band.

4. Applications. - In this section we will briefly describe some of the possible applications of the method just described. The continued fraction expansion method, quite recent, is very powerful for disordered systems and its applicability is just at the beginning. The method needs a precise knowledge of the low order moments of the density of states, and hence a good description of the local order characterizing the random system. Two complementary ways of 
using the method can be used, either by an analytical approach to the study of the moments, or by a purely numerical computation using a simulation of the random system on a computer.

This last approach seems to be particularly useful for random systems with topological disorder, such as amorphous materials or liquid metals, where only limited information on the short range order is known through the pair correlation function.

4.1 Disordered Alloys. - A narrow band random substitutional alloy, made up of two constituents $A$ and $\mathrm{B}$, can be described by a tight binding hamiltonian of the form

$$
\mathscr{H}=\sum_{i} \varepsilon_{i} c_{i}^{+} c_{i}+\sum_{i \neq j} t_{i j} c_{i}^{+} c_{j}
$$

where $\varepsilon_{i}= \pm \frac{1}{2} \delta$ is the atomic level of an atom at site $i$, $t_{i j}$ the transfer matrix between sites $i$ and $j$, and $c_{i}$ an annihilation operator for electrons. The second summation is taken over nearest neighbours only and often $t_{i j}$ is supposed to be periodic, the only random elements of the hamiltonian being the diagonal ones $\varepsilon_{i}$.

For an alloy with a tree (or Bethe) lattice, that is, a lattice with no closed paths (see Fig. 3), the term $a_{0}$ in the continued fraction (eq. (12) and (13)) depends only on the central atom, $b_{0}$ describes a hop from the

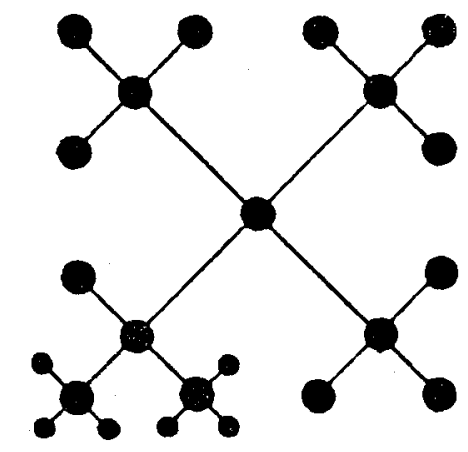

FIG. 3. - A portion of a tree lattice of coordination number $Z=4$.

central atom to the nearest neighbours, $a_{1}$ depends on the nearest neighbours, and so on. By treating the central atom exactly and assuming that an electron hops from the central atom to the average environment, it can be shown [9] that one recovers the usual CPA through the continued fraction technique. Let us here recall briefly in 4.1.1 the derivation given by Jacobs [9] and show how this technique provides a very useful tool to derive results extending the CPA one. In 4.1.2, we will give short indications about the possible numerical approach using a simulation technique.

4.1.1 Continued fraction expansion and the CPA and its extensions. - According to Jacobs [9], it is quite easy to see how the continued fraction expan- sion method leads to the usual CPA equations by assuming that the central atom is treated exactly but the environment is treated in the average.

Indeed, for an ordered tree lattice of coordination number $Z$, i. e. where $\delta=0$ and $t_{i j}=t$ in (17), the diagonal element of the Green function given through eq. (12) can be written in the form of a simple continued fraction $[10,11]$ :

$$
\begin{aligned}
G_{00}(E) & =\frac{1}{E-Z t^{2} F(E)} \\
F(E) & =\frac{1}{E-(Z-1) t^{2} F(E)}
\end{aligned}
$$

Eq. (18a) for $G_{00}(E)$ includes all tree-like chains with no closed loops and $Z-1$ appears in the denominator of $F(E)$ (eq. $(18 b)$ ) because a step back to atom zero would give a reducible chain which is already counted in $(18 a)$.

Considering now the case of a hamiltonian with diagonal disorder $\left(\delta \neq 0, t_{i j}=t\right)$ describing an alloy of concentration $X$, we shall write that the central atom is treated exactly but the environment is treated in the average. If for example, the central atom is of type A, we find in place of eq. (18):

$$
G_{00}^{\mathrm{A}}(E)=\frac{1}{-\frac{1}{2} \delta+E-Z t^{2}-\bar{F}}
$$

with

$$
\begin{aligned}
& \bar{F}(E)=\frac{X}{-\frac{1}{2} \delta+E-(Z-1) t^{2} \bar{F}(E)}+ \\
& +\frac{1-X}{\frac{1}{2} \delta+E-(Z-1) t^{2} \bar{F}(E)} \\
& \vec{F}(E)=X F_{\mathrm{A}}(E)+(1-X) F_{\mathrm{B}}(E)
\end{aligned}
$$

$G_{00}^{\mathrm{B}}(E)$ is given by a similar equation. The appropriate solution to this cubic equation $(19 b)$ gives the average diagonal matrix element of the Green function

$$
\left\langle G_{00}(E)>=X G_{00}^{\mathrm{A}}(E)+(1-X) G_{00}^{\mathrm{B}}(E) .\right.
$$

If the coordination number $Z$ tends to infinity and $t$ is renormalized to $t / Z^{1 / 2}$ to keep a finite bandwidth, then eq. (19b) leads to the usual solution of the CPA for $\left\langle G_{00}(E)\right\rangle[12]$.

Using the mathematical techniques just developed and the principle that the central atom and its local environment, $i$. e. the effect of the first shell of its nearest neighbours, should be treated exactly and the environment of other atoms in an average approximation, we can obtain the site diagonal Green's function of a cluster containing $z_{\mathrm{A}}$ and $z_{\mathrm{B}}$ nearest neighbours around one A (or one B) through eq. (19). This leads to equations similar to those obtained in a Bethe-Peierls approximation (BPA) of a random alloy [13]. This can be understood easily as the BPA is the exact solution for the Ising model on a Cayley 
tree [10]. This approximation leads to a much improved density of states over the CPA result (see for example Fig. 4 and Ref. [13]). The continued fraction expansion method can then be of great use to a better understanding of the nature of the various possible extensions to the CPA [14].

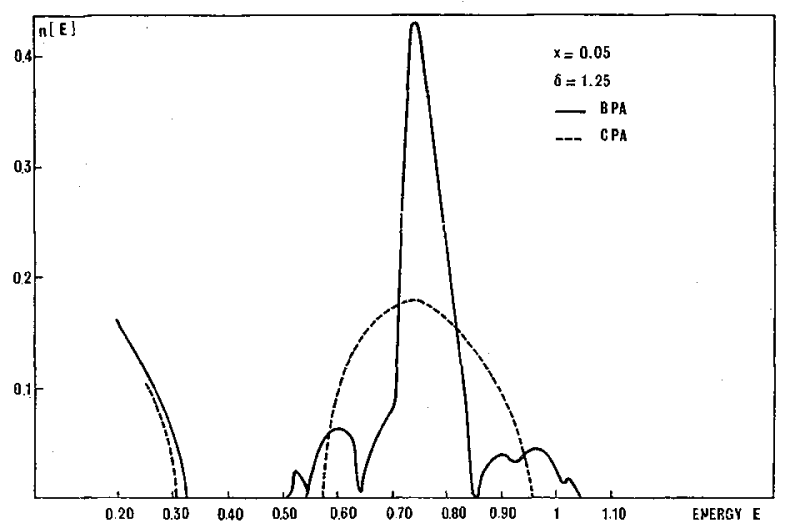

FIG. 4. - CPA and BPA impurity band in the split band limit case (large $\delta$ ).

In the same way, one can deal with the case where the disorder is not confined to the diagonal elements of the hamiltonian, introducing the values $t_{\mathrm{AA}}$, $t_{\mathrm{AB}}, t_{\mathrm{BA}}$ or $t_{\mathrm{BB}}$ depending on the nature of sites $i$ and $j$. The equations can be obtained immediately through eq. (19) [9]. Great simplification occurs when making the assumption that

$$
\begin{aligned}
& t_{\mathrm{AB}}=t_{\mathrm{BA}}=t \\
& t_{\mathrm{AA}}=\alpha t \\
& t_{\mathrm{BB}}=\alpha^{-1} t .
\end{aligned}
$$

Calculations have been carried by Jacobs [9] in the limit $Z \rightarrow \infty$ and $t \rightarrow t / Z^{1 / 2}$ and with various values of parameters. Figure 5 shows an example of the density of states obtained.

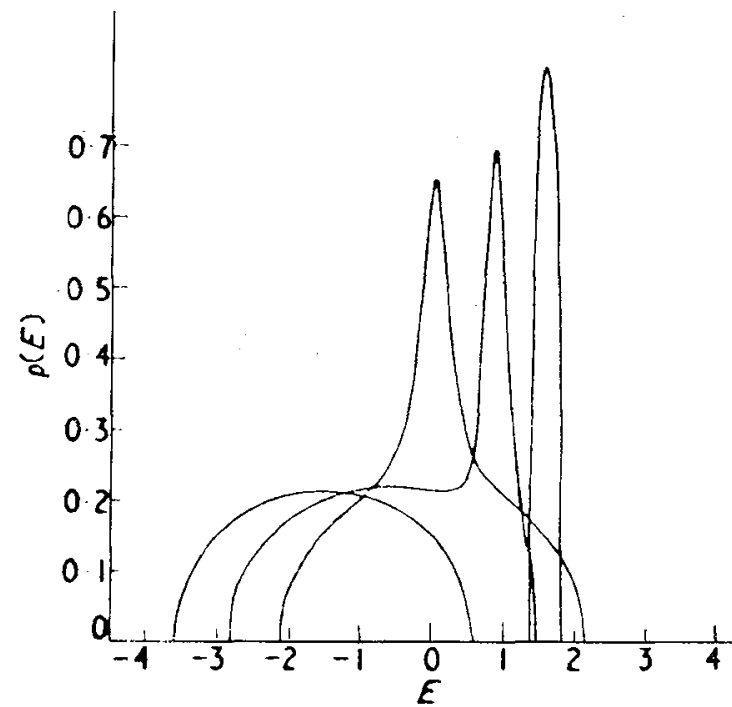

FlG. 5. - Density of states obtained through an extension of the CPA to off-diagonal disorder by Jacobs [9]

$$
x=0.3, \alpha=0.4 \text { and } \delta=0,1.5 \text { and } 3.0 \text {. }
$$

4.1.2 Numerical approach. - The continued fraction expansion technique can be used fruitfully for testing the various possible approximations just briefly sketched through direct numerical computation of the moments. This can be achieved either by a simulation of the random alloy on a computer or by taking into account exactly, for example, the 13 first moments to test the pair approximation. This type of work has been just recently developed $[8,15,16]$ and needs further progress to be of precise use.

4.2 ImPURITy BANDS AND LIQUID METALS. - A transition liquid metal or an impurity band, i. e. positionaly disordered systems case, can be described using a tight binding hamiltonian such as (1), but where the random part is the off-diagonal elements $t_{i j}$ which vary as a function of the distribution of the atoms in the liquid, the diagonal part $\varepsilon$ being assumed independent of the site. The moment method can thus be used to study these systems, but as already emphasized, one needs a good knowledge of the local order characterizing the liquid through the successive correlation functions. This is not the case as only very partial information on the local order can be obtained through the pair correlation function.

Most of the present studies in this field have been done in schematic models, far from representing a realistic liquid metal, such as the random or the hard sphere liquid. Even in the schematic hard sphere liquid, one is usually obliged to make further various simplifying assumptions concerning the high order correlation function which can perhaps explain some of the discrepancies between the results of the various approaches $[12,18,19]$. In this context, a very interesting approach in progress now [8] uses a calculation of the moments of the density of states through a simulation of the liquid on a computer. Furthermore, there is no fundamental impossibility to refine the

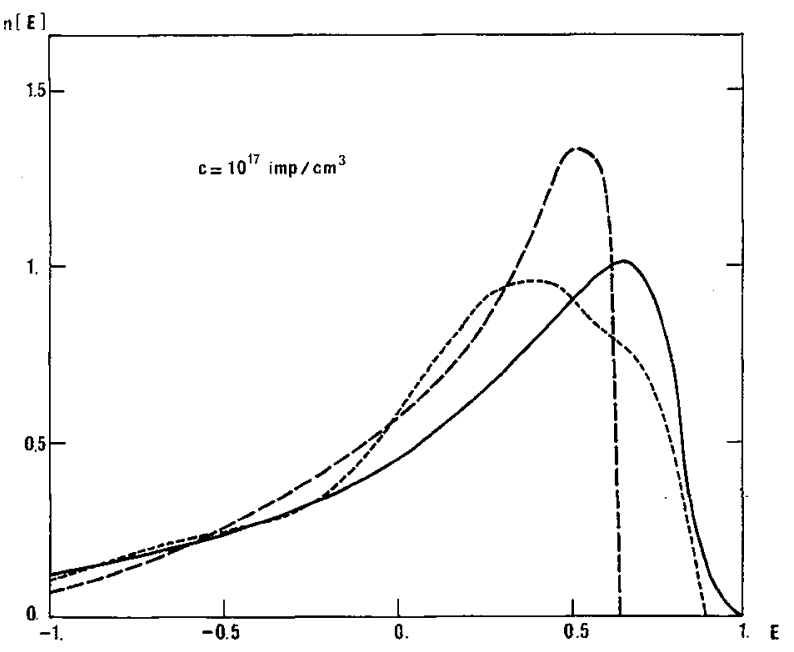

Fig. 6. - Density of states of the impurity band obtained for $\lambda=0.1 ; \longrightarrow$ cumulant approximation $[19] ;-\ldots-$ Matsubara et al. $[20]$; . . . simulation 4$]$. 
hard sphere liquid model by ensuring better forces between atoms so as to reproduce the experimental information known on true liquid metals, but this would involve quite a formidable numerical task.

Apart from its conceptual interest, completely randomly located atoms, the random liquid case is of physical interest as it represents a good approach to the case of an impurity band formed in a heavily doped semiconductor in the range of concentration of metallic conduction [4]. Two approaches to this problem can be made, an analytical one using various approximations to the usual Green's function diagrammatic expansion or equivalently to the calculation of the moments, or a numerical one where the random impurities are simulated on a computer. A detailed comparison has been made for various concentrations of impurities analyzing the discrepancies in the results obtained through the various approaches, especially in the tails of the band [19] due to the effect of fluctuations (see for example Fig. 6). This gives a good idea of the possibilities of the moment method when associating both analytical and numerical approaches.

\section{References}

[1] Lieb, E. H. and Mattis, D. C., «Mathematical Physics in One Dimension» (Acad. Press) 1966.

[2] Cyrot-Lackmann, F., J. Phys. C 5 (1972) 300.

[3] Fisher, M. E. and CAMP, W. J., Phys. Rev. B 5 (1972) 3730.

[4] Gaspard, J. P. and Cyrot-Lackmann, F., J. Phys. C (Solid State Physics) 6 (1973) 3077.

[5] Cyrot-LackmanN, F., J. Phys. C 3 (1972) 167.

[6] Shohat, J. A. and Tamarkin, J. D., "The Problem of Moments », Math. Surv. (A. M. S. Providence) 1963.

[7] Haydock, R, Herne, V. and Kelly, M. J., J. Phys. C (Solid State Physics) 5 (1972) 2845.

[8] Gaspard, J. P., J. Physique 35 (1974) C 4-127.

[9] J JCOBS, R. L., J. Phys. F (Metal Physics) 3 (1973) 933.

[10] Dомв, C., Adv. Phys. 9 (1960) 145.

[11] Brinkman, W. F. and Rice, T. M., Phys. Rev. B 2 (1970) 1324.
[12] VelickỸ, B., Kirkpatrick, S. and Ehrenreich, H., Phys. Rev. 175 (1968) 747.

[13] Brouers, F., Cyrot, M. and Cyrot-Lackmann, F., Phys. Rev, B 7 (1973) 4370.

[14] Cyrot-Lackmann, F., to be published.

[15] Kirkpatrick, S., J. Phys. C 3 (1972) 247.

[16] Mookerjee, A., J. Phys. $C$ (Solid. State Physics) 6 (1973) 1340.

[17] Roth, L. M., Phys. Rev. B 7 (1973) 4321 and J. Physique 35 (1974) C4-317.

[18] Ishida, Y. and Yonezawa, F., Progr. Theor. Phys. 49 (1973) 731.

[19] Crrot-Lackmann, F. and Gaspard, J. P., J. Phys. C (Solid State Physics), to be published.

[20] Matsubara, T. and Toyozawa, Y., Progr. Theor. Phys. 26 (1961) 739. 